\title{
Financial Wellbeing and Mental Health: A Systematic Review
}

\author{
MOHD FAIZUL HASSAN ${ }^{1}$, NAFFISAH MOHD HASSAN ${ }^{2}$, ERNE SUZILA KASSIM ${ }^{3}$, \\ YAHYA MAHYUDDIN UTOH SAID ${ }^{4}$ \\ 1, 2,3, Universiti Teknologi MARA, Cawangan Selangor, Kampus Puncak Alam, MALAYSIA. \\ ${ }^{4}$ Federal Territory Islamic Religious Department, JAWI, MALAYSIA. \\ ${ }^{1}$ E-mail: faizulhassan@uitm.edu.my \\ 2E-mail: naffi885@uitm.edu.my \\ ${ }^{3}$ E-mail: ernekassim@uitm.edu.my \\ [E-mail: mahyuddin@jawi.gov.my
}

\begin{abstract}
The aim of this study is to identify and appraise literatures on relationships between financial well-being and mental health. Conducting a systematic review on what is available from the literature is fundamental as a mean for keeping track of the study progress and synthesizing the emerging setting. The search was performed using related keywords of the two constructs from Psychinfo, Scopus and Google Scholar databases between the year 2001 and 2019. The initial search yields a combination of 2,222 papers and after performing the exclusion criteria, a total of 37 documents were used as the data. Analyses of the studies were conducted to answer the questions of what are the measures of financial wellbeing and mental health and how does financial wellbeing affect mental health? The findings suggest a diverse perspective of financial wellbeing and the term financial hardship, financial situation, financial strain, financial stress and financial security that carry similar meaning were used in literature. There were also various indicators that measure mental health and the use of CES-D, Brief Symptom Inventory (BSI), general health questionnaire (GHQ-28), Mental Health Index (MHI-5), International Diagnostic Interview (WHO-CIDI), Rosenberg Self-Esteem Scale, Goldberg Depression and Anxiety Scales, Depression Anxiety Stress (DASS-21) and Kessler 10 Measure of Psychological Distress (K10) was common. There is an increasing trend of the study and also consistent findings that indicate for significant relationship between financial wellbeing and mental health. Hence, taking a proactive prevention to keep a healthy financial wellbeing such as financial educating and financial literacy is very crucial to reduce the burden of mental depression, and further the burden of healthcare and social protection.
\end{abstract}

Keywords: mental health, financial wellbeing, financial hardship, depression, poverty and stress

JEL Classification: C1, L2 


\section{Introduction}

Depression, anxiety and stress are common mental health issues and have become the world's most pressing public health concerns. Data from the Global Health Data Exchange suggests the state of poor mental health problem is growing, with 17.3 percent or approximately 84 million people in Europe are suffering from the mental health challenge. Depression, anxiety, and stress are symptoms of poor mental health with depression and anxiety become the two most common mental health disorders in community (Organization, 2017). Likewise, a great concern of the public health is the rise of the anxiety and mood disorders (Greenglass et al., 2015). Meanwhile, a finding by (Fiksenbaum et al., 2017) also reported that $3.6 \%$ of people worldwide show the symptom of anxiety disorders, and $4.4 \%$ are suffering from depressive disorder. The mental health problem can lead to premature mortality and affects the quality of life. Research have also found mental health problems are among the most important contributors to the burden of other diseases and disability. Mental illness is a major source of loss of productivity and wellbeing. Losing the mental stability is additional burden to the economic development.

Many factors explain for mental health such as psychosocial stress (Alyousef, 2019), work related factors (Mo et al., 2020) and socio-economic factors (Anderson et al., 2018). Yet, statistics have shown for a consistent link between how the mental condition is affected by the status of financial wellbeing. As the world is facing serious issues of poverty, income instability and poor economic status and global financial crisis, more and more people are trapped in the financial stress situations. Data from the World Health Organization (WHO) indicates there is a steady rise in the percentage of individuals suffering from the financial hardship. This has triggered some worries on the financial burden and explained for today's mental health crisis.

Nowadays, the attention toward the personal financial wellbeing is increasing. Generally financial wellbeing is referring to person's ability to manage the current situation and also ongoing of financial obligations. It is how people feel secure about their financial matters at present or in the future, as the financial ability to make selections for them to enjoy life (Bureau, 2017). In fact, financial wellbeing is associated with financial literacy and financial behaviour. In the same way financial behaviour is one of the components that relates to financial satisfaction or financial wellbeing (Gerrans et al., 2014; Xiao et al., 2014). Likewise, financial wellbeing is broadly defined as the concept used to explain an individual's or a family's financial situation (Mokhtar et al., 2015). It is a subjective sentence towards person's financial status as in condition of zero burden, financially happy and financial healthy (Sabri \& Zakaria, 2015). Certainly, financial wellbeing also defines the perception of people's regarding their financial knowledge and the way of controlling money and monetary value (De Witte et al., 2015).

While there are studies that examine for the relationship between financial wellbeing and mental health, attempts to explore the literature on the association is still lacking. Conducting a systematic review on what is available from the literature is fundamental as a mean for keeping track of the study progress and synthesizing the emerging setting. Therefore, the study was conducted to answer the following research questions, "what are the measures of financial wellbeing and mental health" and how does financial wellbeing affect mental health?

\section{Research Methodology}

The study has undertaken a systematic review of literature, known as a research synthesis for answering the research questions. Research synthesis and systematic literature reviews have gained increasing acceptance within social science research. It is aimed to overcome the issue of researcher bias by using a comprehensive search and analysis framework that combines cross-referencing between researchers, extensive searches of research databases and the application of exclusion criteria (Hedges \& Cooper, 2009). Based on Cooper and Hedges (2009), there are six stages that govern a research synthesis; 1 ) define the problem, 2) collect the research evidence, 3) evaluate of the evidences for inclusion and exclusion (selection criteria), 4) analyze or integrate the evidences, 5) interpret the cumulative evidence and 6) present the synthesis methods and results. The main aim of 
the paper is to provide the understanding of the indicators used to measure financial wellbeing and mental health, and to understand how financial wellbeing affects mental health.

The document retrieval started by searching for the evidences from Scopus-indexed journals, Google Scholar and Psychinfo that include both journals and conference proceedings. The following keywords were used to search for the article: "Financial well-being" "Indebtedness" or "Debt" and „Health" or "Mental disorder" or "Mental illness" or „Depression " or „Anxiety" or „Stress "or „Distress" or "Mental Health". The words regarding the definition term of financial wellbeing with synonyms words were also identified. Although mental disorder is a very broad term, the scope is limited within the impact towards feelings such as distress or behaviors that relate to impairment of function. Table 1 illustrates the summary of the search strategy.

Table 1 Search Strategy for keyword

Terms that were used as a searching strategy for financial wellbeing (combined using OR)

Terms that were used as a search strategy for mental health (combined using OR)

The initial search produced a total of 2,222 documents. As suggested by (Tanskanen et al., 2017), the articles require further evaluations for inclusion and exclusion criteria. The inclusion and exclusion criteria are shown in Table 2.

Table 2 The inclusion and exclusion criteria

\begin{tabular}{|l|l|l|}
\hline Criterion & \multicolumn{1}{|c|}{ Eligibility } & \multicolumn{1}{c|}{ Exclusion } \\
\hline $\begin{array}{l}\text { Literature } \\
\text { type }\end{array}$ & Journal (research articles) & $\begin{array}{l}\text { Journals (systematic review), book series, book, } \\
\text { chapter in book, conference proceeding, papers } \\
\text { did not present primary empirical data (e.g., } \\
\text { commentaries, news reports) }\end{array}$ \\
\hline Language & English & Non-English \\
\hline Time line & Between 20010-2019 & $<2010$ \\
\hline Indexes & $\begin{array}{l}\text { Social Science Citation Index, Emerging } \\
\text { Sources Citation Index, Art and Humanities } \\
\text { Index (Web of Science) }\end{array}$ & \\
\hline
\end{tabular}

Figure 1 The Process of Article Selection

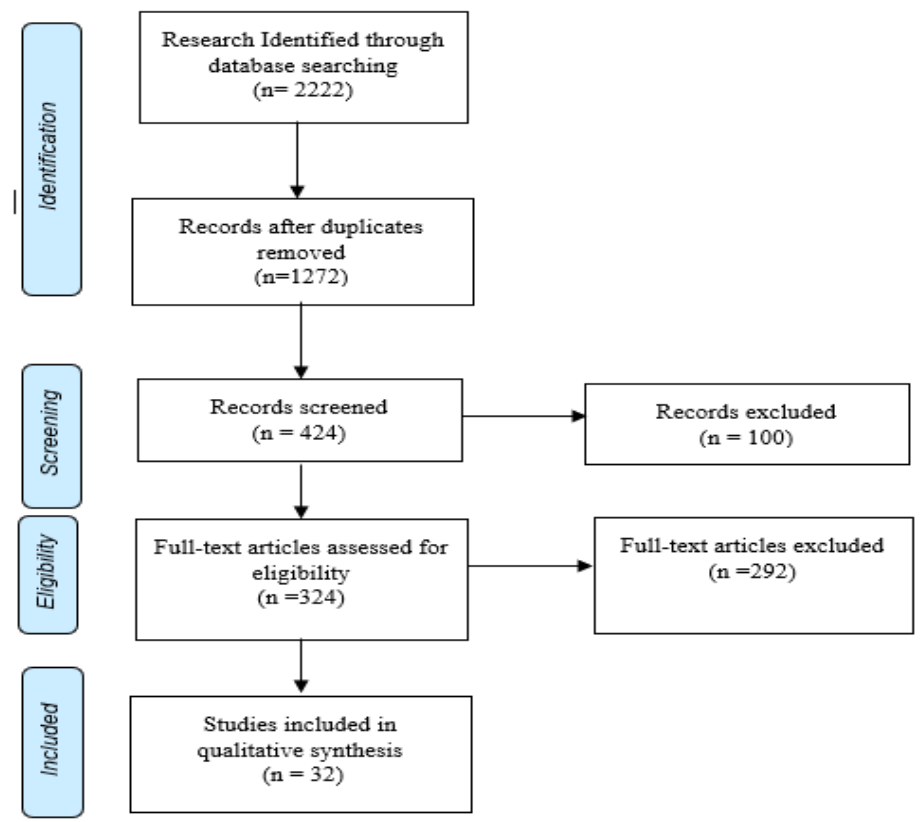


The following diagram illustrates the process of identifying, screening and selecting the articles. After a careful deliberation and confirmation, a total of 32 articles were used as the data.

\section{Results and Findings}

The search strategy yielded 1272 results once duplicates were excluded. Most studies were conducted as surveys. Various indicators were used to measure both financial wellbeing and mental health that answer for the first question. Table 3 and Table 4 provide the list of the measurement tool. The common scales used to measure financial wellbeing are financial hardship, financial strain and personal economic distress. Financial hardship was used in studies conducted by (Butterworth et al., 2012) in a cross-sectional study of 8,841 participants aged 16-85 in Australia's national mental health and wellness survey and Kiely et al., (2015) also in Australia, but on the setting of income and labor dynamics. Financial strain was examined by (Selenko \& Batinic, 2011) on a cross sectional study of clients of a national non-profit, publicly funded Austrian debt-counseling institution who faced bankruptcy, (Thinagaran et al., 2017) who conducted a survey in Malaysia on low-cost flats residents whose household income is within the poverty line.

Table 3 Measurement tools of Financial Wellbeing

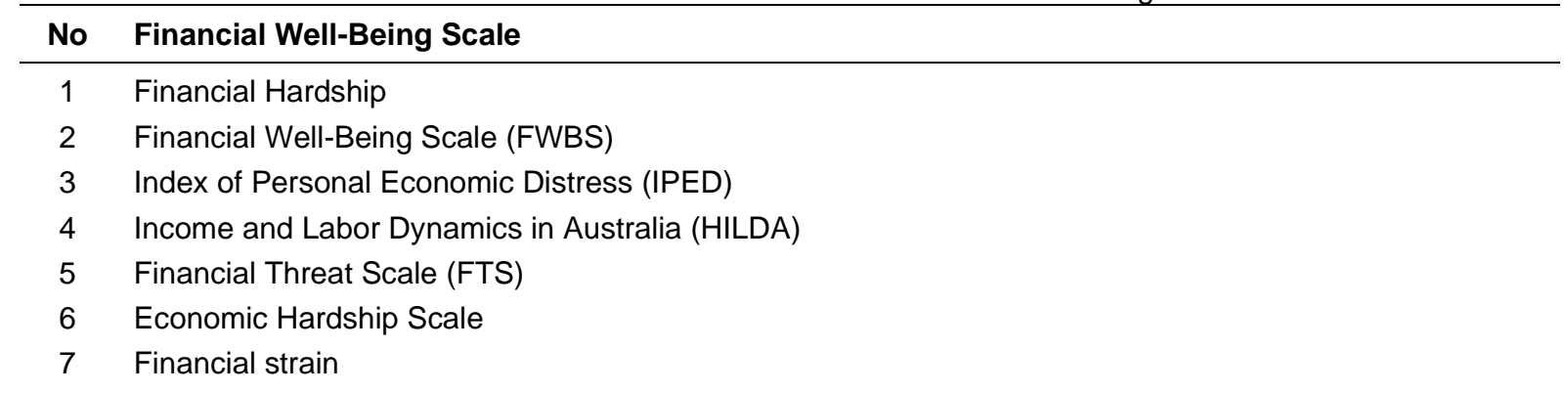

In identifying the similar term of mental health measurement, seven studies were found to use the general health questionnaire (GHQ) in which it is a scale to measure the current mental health, developed by Goldberg (1970). The original GHQ his a 60 -item instrument, but as studies in mental health evolve, efforts were made to simplify and shorten the general health questionnaire, that include versions of GHQ-30, GHQ-28, GHQ-20 and GHQ-12. Other scales which are common to measure mental health are Kassler Psychological Distress Scale, Mental health inventory MH-5 and Structured Clinical Interview etc.

Table 4 Measurement tools of Mental Health

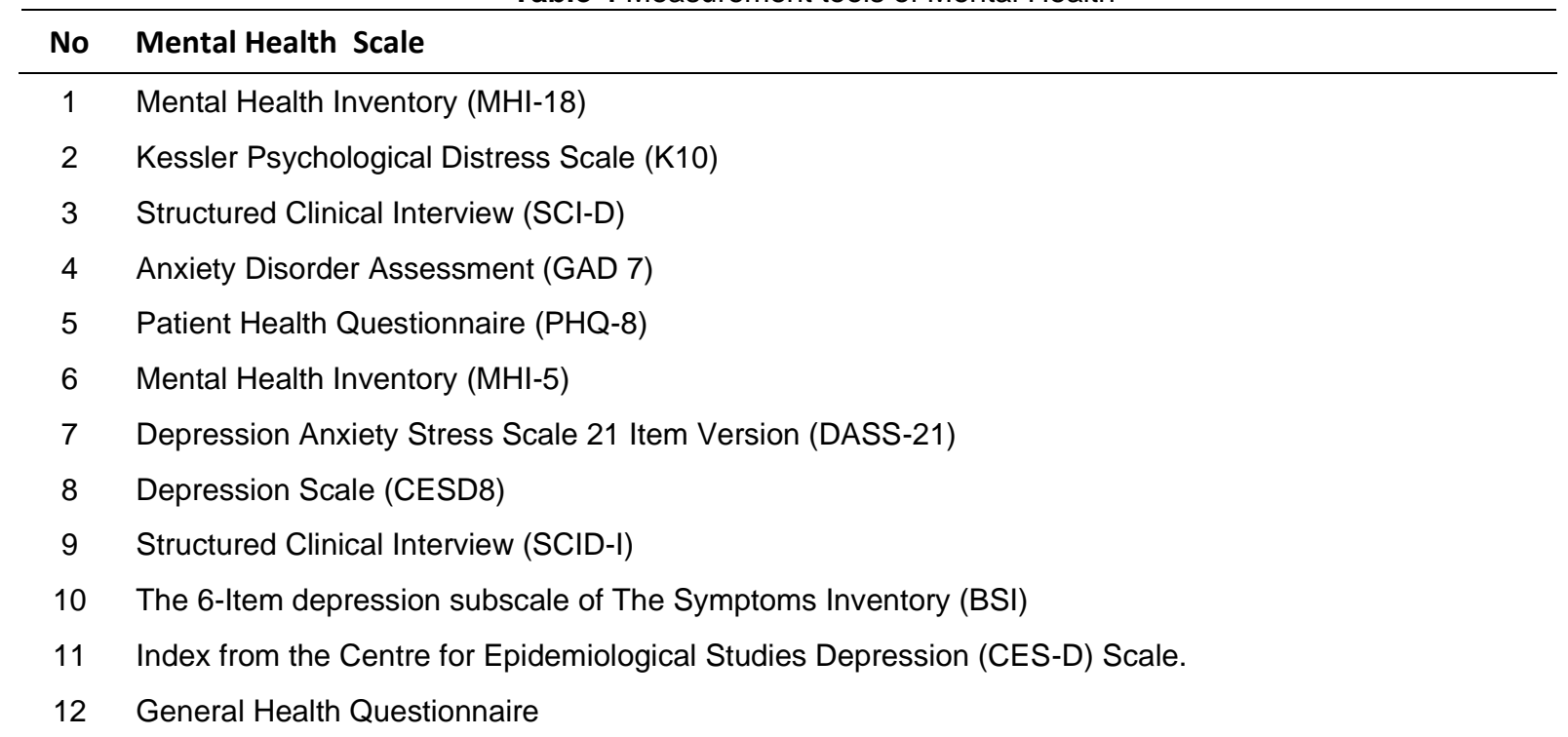




\subsection{Financial Hardship and Mental Health}

This section answers the question of how does financial wellbeing affect mental health? In assessing the relationship between financial wellbeing and mental health, a summary of the studies is presented in Table 5. As there are different scales and measures, yet each of them is measuring the similar concept, the discussion will be based on the specific term and scale. To begin, the assessment was conducted on the relationship between financial hardship and mental health. It was found there are three studies conducted. (Kiely et al., 2015) concluded that current hardship was associated with the increased risk to the mental health problems. Similarly, the study conducted by (M. Taylor et al., 2017) revealed financial hardship factors formed as the strongest associations with reported psychological distress among Western Sydney parents, particularly those factors related to mortgage/housing and employment security. On the other hand, (Economou et al., 2018) agreed that financial hardship was found to be risk factors for major depression.. (Butterworth et al., 2011) used longitudinal data to investigate whether the benefits of having a job depend on its psychosocial quality (levels of control, demands and complexity, job insecurity, and unfair pay) and whether poor quality jobs are associated with better mental health than unemployment. The findings on financial hardship were more strongly associated with depression than other socio-economic variables.

Next, the assessment on the relationship between financial strain and mental health suggests for consistent trend. (Aranda \& Lincoln, 2011) examined the relationship between financial strain and depressive symptoms in later life, and potential psychosocial mediators of this relationship to test the direct effect of chronic financial strain on depressive symptomatology. The findings indicated that financial strain increased the level of depressive symptoms among this sample. (Zürcher et al., 2019) collected data from 511 individuals with $\mathrm{SCl}$ aged over 16 years who participated in the community survey of the Swiss SCl Cohort Study (SwiSCl), and observed an association between Financial strain was associated with general mental health problems and depressive. (Tøge, 2016) used longitudinal data and methods to investigate whether the effect of unemployment on self-rated health (SRH) is mediated by income, financial strain and unemployment benefits. The findings gave the evidences that financial strain is a potential mediator of the individual health effect of unemployment. (Thinagaran et al., 2017) identified that the financial strain has a positive influence by financial behavior and has a negative influence on personal well-being especially to the urban poor community.

Furthermore, using the assessment of financial stress, it was found four studies were within the investigation. (Mandaliya et al., 2016) who conducted a cross-sectional study that involved 65 oncology outpatients found financial stress was not only significantly associated with the cancerspecific quality of life-related issues, but also family-related anxiety, and recurrence-related distress. Meanwhile (Sturgeon et al., 2016) highlighted that financial stress demonstrates the complex relations with the inflammation effects of the psychological wellbeing. This study is similarly with the findings by (Åslund et al., 2014) who found for significant interactions between financial stress, particularly in relation to low psychological well-being.

Table 5 Characteristic of included studies

\begin{tabular}{|c|c|c|c|c|c|c|c|}
\hline Authors & Year & $\begin{array}{l}\text { Study } \\
\text { Design }\end{array}$ & Method & Mental Health & $\begin{array}{c}\text { Financial } \\
\text { Well - Being }\end{array}$ & Conclusion & $\begin{array}{l}\text { Relationship } \\
\text { between } \\
\text { Financial } \\
\text { Wellbeing and } \\
\text { Mental Health } \\
\text { issues }\end{array}$ \\
\hline $\begin{array}{l}\text { (Wilkinso } \\
\text { n, 2016) }\end{array}$ & 2016 & $\begin{array}{l}\text { Cross } \\
\text { Sectional } \\
\text { survey }\end{array}$ & $\begin{array}{l}\text { This research } \\
\text { is concentrated } \\
\text { on } 5,366 \\
\text { respondents } \\
\text { from the Health } \\
\text { and Retirement } \\
\text { Study (2006- } \\
2010) \\
(\mathbf{n = 5 , 3 6 6 )}\end{array}$ & $\begin{array}{l}8 \text { item index of } \\
\text { the Center for } \\
\text { Epidemiologic } \\
\text { al Research on } \\
\text { Depression } \\
\text { (CES-D) of the } \\
\text { scale. }\end{array}$ & $\begin{array}{l}\text { (a) labour } \\
\text { force } \\
\text { participation, } \\
\text { (b) household } \\
\text { income, } \\
\text { (c) financial } \\
\text { wealth, and } \\
\text { (d) net home } \\
\text { equity. }\end{array}$ & $\begin{array}{l}\text { The } \\
\text { financial } \\
\text { resources } \\
\text { associated } \\
\text { with } \\
\text { increased } \\
\text { financial } \\
\text { strain }\end{array}$ & $\begin{array}{l}\text { Recession not } \\
\text { only affected } \\
\text { older adults' } \\
\text { financial well- } \\
\text { being but also } \\
\text { adversely } \\
\text { affected mental } \\
\text { health }\end{array}$ \\
\hline
\end{tabular}




\begin{tabular}{|c|c|c|c|c|c|c|c|}
\hline Authors & Year & $\begin{array}{l}\text { Study } \\
\text { Design }\end{array}$ & Method & Mental Health & $\begin{array}{c}\text { Financial } \\
\text { Well - Being }\end{array}$ & Conclusion & $\begin{array}{l}\text { Relationship } \\
\text { between } \\
\text { Financial } \\
\text { Wellbeing and } \\
\text { Mental Health } \\
\text { issues }\end{array}$ \\
\hline $\begin{array}{l}\text { (Aranda } \\
\& \\
\text { Lincoln, } \\
2011 \text { ) }\end{array}$ & 2011 & $\begin{array}{l}\text { Cross } \\
\text { Sectional } \\
\text { survey }\end{array}$ & $\begin{array}{l}\text { Sample of } 214 \\
\text { low-income } \\
\text { Latino adults } \\
\text { using structural } \\
\text { equation } \\
\text { modeling to } \\
\text { test the direct } \\
\text { effect of } \\
\text { persistent } \\
\text { financial strain } \\
\text { on depressive } \\
\text { symptoms. } \\
\text { (n= 214) }\end{array}$ & \begin{tabular}{|l|} 
The 6 -item \\
depression \\
subscale of the \\
Brief Symptom \\
Inventory (BSI)
\end{tabular} & financial strain & $\begin{array}{l}\text { Financial } \\
\text { strain } \\
\text { increased } \\
\text { the level of } \\
\text { depressive } \\
\text { symptoms } \\
\text { among the } \\
\text { respondents }\end{array}$ & $\begin{array}{l}\text { The differential } \\
\text { effect of financial } \\
\text { stress and coping } \\
\text { styles on } \\
\text { psychological } \\
\text { well-being and } \\
\text { the future } \\
\text { psychosocial } \\
\text { objectives of } \\
\text { treatments for } \\
\text { older adults with } \\
\text { chronic health } \\
\text { conditions. }\end{array}$ \\
\hline $\begin{array}{l}\text { (Lewko } \\
\text { et al., } \\
2019)\end{array}$ & 2019 & $\begin{array}{l}\text { Cross } \\
\text { Sectional } \\
\text { survey }\end{array}$ & $\begin{array}{l}\text { The study was } \\
\text { conducted in a } \\
\text { group of } 523 \\
\text { randomly } \\
\text { selected } \\
\text { professionally } \\
\text { active nurses } \\
\text { aged over } 40 \\
\text { years old from } \\
\text { the Podlaskie } \\
\text { Voivodeship } \\
\text { (n= 523) }\end{array}$ & $\begin{array}{l}\text { the general } \\
\text { health } \\
\text { questionnaire } \\
\text { (GHQ-28), }\end{array}$ & $\begin{array}{l}\text { satisfaction } \\
\text { with life scale } \\
\text { (SWLS) }\end{array}$ & $\begin{array}{l}\text { Respondent } \\
\text { s' financial } \\
\text { situation } \\
\text { greatly } \\
\text { influenced } \\
\text { their quality } \\
\text { of life and } \\
\text { affected } \\
\text { mental } \\
\text { health } \\
\text { components }\end{array}$ & $\begin{array}{l}\text { The mental } \\
\text { health component } \\
\text { was found to be } \\
\text { significantly } \\
\text { affected by } \\
\text { financial situation }\end{array}$ \\
\hline $\begin{array}{l}\text { (Rajani } \\
\text { et al., } \\
2016)\end{array}$ & 2016 & $\begin{array}{l}\text { Linear } \\
\text { regression }\end{array}$ & $\begin{array}{l}\text { The sample } \\
\text { consisted of } \\
\text { employed } \\
\text { people from } 27 \\
\text { European } \\
\text { countries } \\
\text { where the } \\
\text { Eurobarometer } \\
\text { survey (73.2 } \\
\text { wave, 2010) } \\
\text { was } \\
\text { administered } \\
\text { by the } \\
\text { European } \\
\text { Commission }\end{array}$ & $\begin{array}{l}\text { Mental Health } \\
\text { Index (MHI-5) }\end{array}$ & $\begin{array}{l}\text { job insecurity } \\
\text { and financial } \\
\text { difficulties }\end{array}$ & $\begin{array}{l}\text { This } \\
\text { research } \\
\text { highlights } \\
\text { the negative } \\
\text { effects of } \\
\text { work } \\
\text { instability } \\
\text { and financial } \\
\text { difficulties } \\
\text { on EU } \\
\text { mental } \\
\text { health. } \\
\text { Employee } \\
\text { assistance } \\
\text { facing job } \\
\text { security } \\
\text { issues } \\
\text { should be a } \\
\text { priority, } \\
\text { regardless } \\
\text { of financial } \\
\text { circumstanc } \\
\text { es. }\end{array}$ & $\begin{array}{l}\text { Poor mental } \\
\text { health can also } \\
\text { increase work } \\
\text { insecurity, so the } \\
\text { detected } \\
\text { association may } \\
\text { be bi-directional. }\end{array}$ \\
\hline
\end{tabular}




\begin{tabular}{|c|c|c|c|c|c|c|c|}
\hline Authors & Year & $\begin{array}{l}\text { Study } \\
\text { Design }\end{array}$ & Method & Mental Health & $\begin{array}{c}\text { Financial } \\
\text { Well - Being }\end{array}$ & Conclusion & $\begin{array}{c}\text { Relationship } \\
\text { between } \\
\text { Financial } \\
\text { Wellbeing and } \\
\text { Mental Health } \\
\text { issues }\end{array}$ \\
\hline $\begin{array}{l}\text { (Zürcher } \\
\text { et al., } \\
\text { 2019) }\end{array}$ & 2019 & $\begin{array}{l}\text { Cross } \\
\text { Sectional } \\
\text { data }\end{array}$ & $\begin{array}{l}\text { cross-sectional } \\
\text { data from } 511 \\
\text { individuals with } \\
\text { SCl aged over } \\
16 \text { years who } \\
\text { participated in } \\
\text { the community } \\
\text { survey of the } \\
\text { Swiss SCl } \\
\text { Cohort Study } \\
\text { (SwiSCl). } \\
\text { (n= 511) }\end{array}$ & $\begin{array}{l}\text { Mental Health } \\
\text { Inventory } \\
\text { (MHI-5) }\end{array}$ & $\begin{array}{l}\text { household } \\
\text { income, and } \\
\text { financial strain }\end{array}$ & $\begin{array}{l}\text { This study } \\
\text { provides } \\
\text { evidence of } \\
\text { socioecono } \\
\text { mic } \\
\text { differences } \\
\text { in social } \\
\text { relationships } \\
\text { and } \\
\text { independent } \\
\text { correlations } \\
\text { of financial } \\
\text { strain and } \\
\text { poor social } \\
\text { relationships } \\
\text { in } \\
\text { individuals } \\
\text { with SCl } \\
\text { with mental } \\
\text { health } \\
\text { issues. }\end{array}$ & $\begin{array}{l}\text { Financial strain } \\
\text { was associated } \\
\text { with general } \\
\text { mental health } \\
\text { issues and } \\
\text { depression, but } \\
\text { education and } \\
\text { household } \\
\text { income were not } \\
\text { related to mental } \\
\text { health }\end{array}$ \\
\hline $\begin{array}{l}\text { (Esan et } \\
\text { al., } \\
2012)\end{array}$ & 2012 & $\begin{array}{l}\text { an } \\
\text { epidemio } \\
\text { logical } \\
\text { survey }\end{array}$ & $\begin{array}{l}\text { An } \\
\text { epidemiological } \\
\text { study examined } \\
\text { mental illnesses } \\
\text { and personal } \\
\text { earnings in a } \\
\text { representative } \\
\text { sample of } 1,889 \\
\text { Nigerians aged } \\
18-64 \text { years., } \\
\text { (n= 1,889) }\end{array}$ & $\begin{array}{l}\text { International } \\
\text { Diagnostic } \\
\text { Interview } \\
\text { (WHO-CIDI) }\end{array}$ & $\begin{array}{l}\text { Personal } \\
\text { earnings } \\
\text { before tax in } \\
\text { the past } 12 \\
\text { months }\end{array}$ & $\begin{array}{l}\text { Mental } \\
\text { illnesses } \\
\text { have } \\
\text { significant } \\
\text { negative } \\
\text { effects both } \\
\text { at the } \\
\text { individual } \\
\text { and social } \\
\text { level on } \\
\text { earnings. } \\
\text { This study } \\
\text { highlights } \\
\text { the financial } \\
\text { value of lost } \\
\text { wages } \\
\text { without such } \\
\text { disorders. }\end{array}$ & $\begin{array}{l}\text { Mental illnesses } \\
\text { have a significant } \\
\text { individual and } \\
\text { community } \\
\text { financial burden. } \\
\text { This impact is } \\
\text { more severe in } \\
\text { men }\end{array}$ \\
\hline
\end{tabular}




\begin{tabular}{|c|c|c|c|c|c|c|c|}
\hline Authors & Year & $\begin{array}{l}\text { Study } \\
\text { Design }\end{array}$ & Method & Mental Health & $\begin{array}{c}\text { Financial } \\
\text { Well - Being }\end{array}$ & Conclusion & $\begin{array}{l}\text { Relationship } \\
\text { between } \\
\text { Financial } \\
\text { Wellbeing and } \\
\text { Mental Health } \\
\text { issues }\end{array}$ \\
\hline $\begin{array}{l}\text { (Jones } \\
\text { et al., } \\
2018)\end{array}$ & 2019 & $\begin{array}{l}\text { cross- } \\
\text { sectional }\end{array}$ & $\begin{array}{l}\text { National Health } \\
\text { and Aging } \\
\text { Trends Study } \\
\text { (NHATS). } \\
\text { Participants data } \\
\text { gathered } \\
(\mathbf{n}=\mathbf{3 0 7})\end{array}$ & $\begin{array}{l}\text { Depressive } \\
\text { symptoms } \\
\text { were assessed } \\
\text { using the } \\
\text { Patient Health } \\
\text { Questionnaire } \\
2 \text { (PHQ2). } \\
\text { General } \\
\text { anxiety was } \\
\text { assessed } \\
\text { using the } \\
\text { Generalized } \\
\text { Anxiety } \\
\text { Disorder } 2 \\
\text { (GAD2). } \\
\text { Self-rated } \\
\text { health. Self- } \\
\text { rated health } \\
\text { was assessed } \\
\text { using the } \\
\text { standard single } \\
\text { item measure }\end{array}$ & \begin{tabular}{|l|} 
Financial \\
problem \\
reported in the \\
past year \\
counting \\
toward of the \\
financial \\
problems
\end{tabular} & $\begin{array}{l}\text { The first } \\
\text { survey's } \\
\text { financial } \\
\text { burden } \\
\text { predicted } \\
\text { depressive } \\
\text { symptoms } \\
\text { the first } \\
\text { survey did } \\
\text { not predict } \\
\text { later } \\
\text { financial } \\
\text { burdens } \\
\text { Depressive } \\
\text { symptoms, } \\
\text { general } \\
\text { anxiety, and } \\
\text { self-rated } \\
\text { well-being }\end{array}$ & $\begin{array}{l}\text { Financial support } \\
\text { for the prediction } \\
\text { of depressive } \\
\text { symptoms, } \\
\text { general anxiety } \\
\text { and self-rated } \\
\text { wellbeing have } \\
\text { been found. }\end{array}$ \\
\hline $\begin{array}{l}\text { (Antunes } \\
\text { et al., } \\
2019)\end{array}$ & 2019 & $\begin{array}{l}\text { Cross } \\
\text { sectional }\end{array}$ & $\begin{array}{l}\text { The data } \\
\text { gathered from } \\
\text { the } 3 \text { of primary } \\
\text { health centres in } \\
\text { Lisbon } \\
\text { Metropolitan } \\
\text { Area. } \\
\text { Focus groups } \\
\text { and interviews } \\
\text { with consumers } \\
\text { and experts } \\
\text { were conducted } \\
\text { in a semi- } \\
\text { structured way. } \\
\text { Interview and } \\
\text { orally transcribed } \\
\text { by using } \\
\text { thematically } \\
\text { analysing }\end{array}$ & $\begin{array}{l}\text { Indicators of } \\
\text { mental health } \\
\text { and wellbeing }\end{array}$ & $\begin{array}{l}\text { Financial } \\
\text { situation }\end{array}$ & $\begin{array}{l}\text { This study } \\
\text { provided a } \\
\text { comprehens } \\
\text { ive overview } \\
\text { of the } \\
\text { bidirectional } \\
\text { relationship } \\
\text { between } \\
\text { socioecono } \\
\text { mic } \\
\text { conditions } \\
\text { and mental } \\
\text { health. }\end{array}$ & $\begin{array}{l}\text { Emphasizing the } \\
\text { need to develop } \\
\text { policies to } \\
\text { address the } \\
\text { drivers of poor } \\
\text { mental health } \\
\text { during times of } \\
\text { economic } \\
\text { downturn and } \\
\text { tackle the barriers } \\
\text { faced by people } \\
\text { with mental } \\
\text { disorders to } \\
\text { promote their full } \\
\text { social inclusion. }\end{array}$ \\
\hline $\begin{array}{l}\text { (Econom } \\
\text { ou et al., } \\
2016)\end{array}$ & 2016 & $\begin{array}{l}\text { Cross- } \\
\text { sectional }\end{array}$ & $\begin{array}{l}\text { A random and } \\
\text { representative } \\
\text { sample of } 2,188 \\
\text { people was } \\
\text { interviewed } \\
\text { by telephone } \\
\text { about various } \\
\text { socio-economic } \\
\text { measures, major } \\
\text { depression and } \\
\text { suicidality } \\
(\mathbf{n = 2 1 8 8 )}\end{array}$ & $\begin{array}{l}\text { Structured } \\
\text { Clinical } \\
\text { Interview } \\
\text { (SCID) }\end{array}$ & $\begin{array}{l}\text { Index of } \\
\text { Personal } \\
\text { Economic } \\
\text { Distress } \\
\text { (IPED), }\end{array}$ & $\begin{array}{l}\text { Economic } \\
\text { hardship } \\
\text { and } \\
\text { unemploym } \\
\text { ent's impact } \\
\text { on } \\
\text { suicidality } \\
\text { were also } \\
\text { significant } \\
\text { and } \\
\text { independent } \\
\text { of major } \\
\text { depression. }\end{array}$ & $\begin{array}{l}\text { Results } \\
\text { emphasize the } \\
\text { urgent need to } \\
\text { design and } \\
\text { implement social } \\
\text { policies and } \\
\text { measures to } \\
\text { mitigate the dire } \\
\text { impact of } \\
\text { Greece's } \\
\text { sustained } \\
\text { recession. }\end{array}$ \\
\hline
\end{tabular}




\begin{tabular}{|c|c|c|c|c|c|c|c|}
\hline Authors & Year & $\begin{array}{l}\text { Study } \\
\text { Design }\end{array}$ & Method & Mental Health & $\begin{array}{c}\text { Financial } \\
\text { Well - Being }\end{array}$ & Conclusion & $\begin{array}{l}\text { Relationship } \\
\text { between } \\
\text { Financial } \\
\text { Wellbeing and } \\
\text { Mental Health } \\
\text { issues }\end{array}$ \\
\hline $\begin{array}{l}\text { (Tøge, } \\
2016)\end{array}$ & 2016 & $\begin{array}{l}\text { Cross- } \\
\text { sectional }\end{array}$ & $\begin{array}{l}\text { This study uses } \\
\text { the European } \\
\text { Union Income } \\
\text { and Living } \\
\text { Statistics (EU- } \\
\text { SILC) panel } \\
\text { 2008-2011 } \\
\text { covering } 28 \\
\text { European } \\
\text { countries (EU-28 } \\
\text { except for } \\
\text { Germany and } \\
\text { Ireland plus } \\
\text { Norway and } \\
\text { Iceland) }\end{array}$ & $\begin{array}{l}\text { General Health } \\
\text { Questionnaire } \\
\text { (GHQ-12) }\end{array}$ & $\begin{array}{l}\text { Individual } \\
\text { fixed effects } \\
\text { models }\end{array}$ & $\begin{array}{l}\text { The } \\
\text { financial } \\
\text { burden is } \\
\text { found to be } \\
\text { a possible } \\
\text { mediator of } \\
\text { the personal } \\
\text { health } \\
\text { effects of } \\
\text { unemploym } \\
\text { ent. In } \\
\text { contrast, } \\
\text { neither total } \\
\text { income, } \\
\text { relative } \\
\text { income, } \\
\text { relative } \\
\text { rank, } \\
\text { income } \\
\text { deprivation, } \\
\text { nor } \\
\text { unemploym } \\
\text { ent benefits } \\
\text { are found to } \\
\text { be } \\
\text { mediators of } \\
\text { that } \\
\text { relationship }\end{array}$ & $\begin{array}{l}\text { Financial strain } \\
\text { as mediators of } \\
\text { the health impact } \\
\text { of unemployment } \\
\text { in the first years } \\
\text { of Europe's } \\
\text { financial crisis }\end{array}$ \\
\hline $\begin{array}{l}\text { Richard } \\
\text { son et } \\
\text { al., } \\
2019 \text { ) }\end{array}$ & 2019 & $\begin{array}{l}\text { Cross } \\
\text { sectional }\end{array}$ & $\begin{array}{l}\text { Fifty-four } \\
\text { participants } \\
\text { diagnosed with } \\
\text { bipolar disorder } \\
\text { in an adult } \\
\text { secondary NHS } \\
\text { mental health } \\
\text { service } \\
\text { completed the } \\
\text { questionnaire } \\
\text { set, which } \\
\text { included steps to } \\
\text { evaluate } \\
\text { financial } \\
\text { variables, } \\
\text { including bill } \\
\text { payment } \\
\text { difficulties and } \\
\text { perceived } \\
\text { financial well- } \\
\text { being. } \\
\text { (n = 54) }\end{array}$ & $\begin{array}{l}\text { Rosenberg } \\
\text { Self-Esteem } \\
\text { Scale } \\
\text { (Rosenberg, } \\
\text { 1989): } \\
\text { Mindful } \\
\text { Attention } \\
\text { Awareness } \\
\text { Scale }\end{array}$ & $\begin{array}{l}\text { Index of } \\
\text { Financial } \\
\text { Stress }\end{array}$ & $\begin{array}{l}\text { Financial } \\
\text { difficulties } \\
\text { contribute to } \\
\text { bipolar } \\
\text { disorder's } \\
\text { mental } \\
\text { health. } \\
\text { Poor mental } \\
\text { wellbeing } \\
\text { leads to } \\
\text { compulsive } \\
\text { buying, } \\
\text { while } \\
\text { financial } \\
\text { concern } \\
\text { raises } \\
\text { anxiety and } \\
\text { stress with a } \\
\text { vicious } \\
\text { anxiety } \\
\text { cycle. }\end{array}$ & $\begin{array}{l}\text { Greater anxiety } \\
\text { and stress were } \\
\text { significantly } \\
\text { linked to higher } \\
\text { compulsive } \\
\text { purchasing } \\
\text { ratings and lower } \\
\text { perceived } \\
\text { financial well- } \\
\text { being. }\end{array}$ \\
\hline
\end{tabular}




\begin{tabular}{|c|c|c|c|c|c|c|c|}
\hline Authors & Year & $\begin{array}{l}\text { Study } \\
\text { Design }\end{array}$ & Method & Mental Health & $\begin{array}{c}\text { Financial } \\
\text { Well - Being }\end{array}$ & Conclusion & $\begin{array}{l}\text { Relationship } \\
\text { between } \\
\text { Financial } \\
\text { Wellbeing and } \\
\text { Mental Health } \\
\text { issues }\end{array}$ \\
\hline $\begin{array}{l}\text { (Mandali } \\
\text { ya et al., } \\
2016)\end{array}$ & 2016 & $\begin{array}{l}\text { Cross } \\
\text { sectional }\end{array}$ & $\begin{array}{l}\text { In this cross- } \\
\text { sectional study, } \\
65 \text { participants } \\
\text { visiting the } \\
\text { outpatient } \\
\text { oncology clinic } \\
\text { obtained a self- } \\
\text { administered } \\
\text { questionnaire. } \\
\text { (n=65) }\end{array}$ & $\begin{array}{l}\text { Standard } \\
\text { Quality of Life } \\
\text { in Adult } \\
\text { Cancer } \\
\text { Survivors } \\
\text { Scale } \\
\text { (QLACS) }\end{array}$ & $\begin{array}{l}\text { Personal and } \\
\text { Household } \\
\text { Finances } \\
\text { (HILDA) }\end{array}$ & $\begin{array}{l}\text { financial } \\
\text { stress was } \\
\text { significantly } \\
\text { associated } \\
\text { with the } \\
\text { cancer- } \\
\text { specific } \\
\text { quality of } \\
\text { life-related } \\
\text { issues, } \\
\text { family- } \\
\text { related } \\
\text { anxiety, and } \\
\text { recurrence- } \\
\text { related } \\
\text { distress. }\end{array}$ & $\begin{array}{l}\text { Most } \\
\text { demographic } \\
\text { characteristics } \\
\text { were not } \\
\text { correlated with } \\
\text { financial stress. }\end{array}$ \\
\hline $\begin{array}{l}\text { (Sturgeo } \\
\text { n et al., } \\
2016)\end{array}$ & 2016 & $\begin{array}{l}\text { Cross } \\
\text { sectional }\end{array}$ & $\begin{array}{l}\text { A group sample } \\
\text { of } 680 \text { middle- } \\
\text { aged adults (age } \\
40-65 \text { ) assessed } \\
\text { stressful } \\
\text { financial and } \\
\text { interpersonal } \\
\text { activities over } \\
\text { the previous } \\
\text { year, perceived } \\
\text { social standing, } \\
\text { indices of } \\
\text { psychological } \\
\text { well-being and } \\
\text { anxiety, and } \\
\text { levels of } \\
\text { interleukin-6 and } \\
\text { C-reactive } \\
\text { protein. } \\
\text { ( } \mathrm{n}=680 \text { ) }\end{array}$ & $\begin{array}{l}\text { Mental Health } \\
\text { Inventory } \\
\text { (MHI-18) }\end{array}$ & $\begin{array}{l}\text { Psychiatric } \\
\text { Epidemiology } \\
\text { Research } \\
\text { Interview } \\
\text { (PERI) Life } \\
\text { Events Scale } \\
\text { (25) }\end{array}$ & $\begin{array}{l}\text { Financial } \\
\text { stress } \\
\text { indicates } \\
\text { complex } \\
\text { inflammatio } \\
\mathrm{n} \\
\text { relationship } \\
\mathrm{s} \text {, partially } \\
\text { due to } \\
\text { psychologic } \\
\text { al well-being } \\
\text { and social } \\
\text { perceptions. }\end{array}$ & $\begin{array}{l}\text { Individuals } \\
\text { experiencing } \\
\text { more significant } \\
\text { financial stressors } \\
\text { over the previous } \\
\text { year showed } \\
\text { more } \\
\text { interpersonal } \\
\text { stressors, greater } \\
\text { psychological } \\
\text { distress, and } \\
\text { lower } \\
\text { psychological } \\
\text { well-being. }\end{array}$ \\
\hline $\begin{array}{l}\text { (Åslund } \\
\text { et al., } \\
2014)\end{array}$ & 2014 & $\begin{array}{l}\text { Cross } \\
\text { sectional }\end{array}$ & $\begin{array}{l}\text { In } 2004 \text { and } \\
2008, \text { two } \\
\text { independent } \\
\text { postal surveys } \\
\text { were } \\
\text { sent to random } \\
\text { samples in five } \\
\text { counties in } \\
\text { Sweden. } \\
\text { (n=84263) }\end{array}$ & $\begin{array}{l}\text { (General } \\
\text { Health } \\
\text { Questionnaire- } \\
\text { 12). }\end{array}$ & $\begin{array}{l}\text { Financial } \\
\text { stress }\end{array}$ & $\begin{array}{l}\text { Social help } \\
\text { had the } \\
\text { most } \\
\text { significant } \\
\text { impact on } \\
\text { high } \\
\text { financial } \\
\text { stress. } \\
\text { Strengtheni } \\
\text { ng social } \\
\text { networks } \\
\text { can } \\
\text { influence } \\
\text { healthcare } \\
\text { costs and } \\
\text { increase the } \\
\text { quality of } \\
\text { life. }\end{array}$ & $\begin{array}{l}\text { Significant } \\
\text { interactions } \\
\text { between financial } \\
\text { stress and social } \\
\text { support, } \\
\text { particularly with } \\
\text { low psychological } \\
\text { well-being. }\end{array}$ \\
\hline
\end{tabular}




\begin{tabular}{|c|c|c|c|c|c|c|c|}
\hline Authors & Year & $\begin{array}{l}\text { Study } \\
\text { Design }\end{array}$ & Method & Mental Health & $\begin{array}{c}\text { Financial } \\
\text { Well - Being }\end{array}$ & Conclusion & $\begin{array}{l}\text { Relationship } \\
\text { between } \\
\text { Financial } \\
\text { Wellbeing and } \\
\text { Mental Health } \\
\text { issues }\end{array}$ \\
\hline $\begin{array}{l}\text { (Econom } \\
\text { ou et al., } \\
2013)\end{array}$ & 2013 & & $\begin{array}{l}\text { In } 2008 \text { and } \\
2011, \text { two } \\
\text { national cross- } \\
\text { sectional } \\
\text { telephone } \\
\text { surveys followed } \\
\text { the same } \\
\text { approach. A } \\
\text { random sample } \\
\text { of } 2,197 \text { and } \\
2,256 \\
\text { individuals, } \\
\text { respectively, } \\
\text { participated in } \\
\text { the studies. } \\
\text { ( } \mathrm{n=4453)}\end{array}$ & $\begin{array}{l}\text { Structured } \\
\text { Clinical } \\
\text { Interview } \\
\text { (SCID-I) }\end{array}$ & $\begin{array}{l}\text { the Index of } \\
\text { Personal } \\
\text { Economic } \\
\text { Distress was } \\
\text { employed } \\
\text { (Madianos et } \\
\text { al., 2011). }\end{array}$ & $\begin{array}{l}\text { A profound } \\
\text { and } \\
\text { detrimental } \\
\text { impact of } \\
\text { the } \\
\text { economic } \\
\text { crisis on the } \\
\text { Greek } \\
\text { population's } \\
\text { mental } \\
\text { health, and } \\
\text { particularly } \\
\text { on the } \\
\text { prevalence } \\
\text { of major } \\
\text { depression. }\end{array}$ & $\begin{array}{l}\text { The economic } \\
\text { crisis' effect on } \\
\text { the population's } \\
\text { mental wellbeing } \\
\text { is widespread. }\end{array}$ \\
\hline $\begin{array}{l}\text { (Butterw } \\
\text { orth et } \\
\text { al., } \\
2012 \text { ) }\end{array}$ & 2012 & $\begin{array}{l}\text { Cross } \\
\text { sectional }\end{array}$ & $\begin{array}{l}\text { Data from 8,841 } \\
\text { participants aged } \\
16-85 \text { in } \\
\text { Australia's } 2007 \\
\text { National Mental } \\
\text { Health and } \\
\text { Wellness } \\
\text { Survey. } \\
\\
\text { (n=8841) }\end{array}$ & $\begin{array}{l}\text { Mental Health } \\
\text { Composite } \\
\text { International } \\
\text { Diagnostic } \\
\text { Interview } \\
\text { (WMH-CIDI } \\
\text { 3.0) }\end{array}$ & $\begin{array}{l}\text { Financial } \\
\text { hardship }\end{array}$ & $\begin{array}{l}\text { The findings } \\
\text { show the } \\
\text { crucial role } \\
\text { of financial } \\
\text { hardship in } \\
\text { the } \\
\text { relationship } \\
\text { between } \\
\text { socio- } \\
\text { economic } \\
\text { disadvantag } \\
\text { e and 12- } \\
\text { month } \\
\text { depressive } \\
\text { episode and } \\
\text { indicate that } \\
\text { social and } \\
\text { economic } \\
\text { policies } \\
\text { addressing } \\
\text { inequality in } \\
\text { living } \\
\text { conditions } \\
\text { could be an } \\
\text { effective } \\
\text { way to } \\
\text { minimize the } \\
\text { burden of } \\
\text { depression. }\end{array}$ & $\begin{array}{l}\text { Financial } \\
\text { hardship was } \\
\text { related to } \\
\text { depression than } \\
\text { other socio- } \\
\text { economic factors. }\end{array}$ \\
\hline
\end{tabular}




\begin{tabular}{|c|c|c|c|c|c|c|c|}
\hline Authors & Year & $\begin{array}{l}\text { Study } \\
\text { Design }\end{array}$ & Method & Mental Health & $\begin{array}{c}\text { Financial } \\
\text { Well - Being }\end{array}$ & Conclusion & $\begin{array}{l}\text { Relationship } \\
\text { between } \\
\text { Financial } \\
\text { Wellbeing and } \\
\text { Mental Health } \\
\text { issues }\end{array}$ \\
\hline $\begin{array}{l}\text { (Sargent } \\
\text {-Cox et } \\
\text { al., } \\
2011 \text { ) }\end{array}$ & 2011 & $\begin{array}{l}\text { Prospecti } \\
\text { ve cohort } \\
\text { study } \\
(20 \mathrm{e} 24, \\
40 \mathrm{e} 44, \\
\text { and } \\
60 \mathrm{e} 65 \\
\text { years at } \\
\text { baseline) }\end{array}$ & $\begin{array}{l}\text { Participants } \\
\text { were drawn from } \\
\text { the PATH } \\
\text { Through Life } \\
\text { Study, a large } \\
\text { longitudinal } \\
\text { cohort study } \\
\text { (20e24, 40e44, } \\
\text { and 60e65years } \\
\text { at baseline) from } \\
\text { the Canberra/ } \\
\text { Queanbeyan } \\
\text { districts, } \\
\text { Australia. }\end{array}$ & $\begin{array}{l}\text { Goldberg } \\
\text { Depression } \\
\text { and Anxiety } \\
\text { Scales }\end{array}$ & $\begin{array}{l}\text { Financial } \\
\text { security }\end{array}$ & $\begin{array}{l}\text { A unique } \\
\text { contribution } \\
\text { to social and } \\
\text { health } \\
\text { literature by } \\
\text { explaining } \\
\text { the physical } \\
\text { and mental } \\
\text { health } \\
\text { consequenc } \\
\text { es of older } \\
\text { adults }\end{array}$ & $\begin{array}{l}\text { A substantial } \\
\text { increase in } \\
\text { symptoms of } \\
\text { depression and } \\
\text { anxiety during the } \\
\text { GFC (Global } \\
\text { Financial Crisis) } \\
\text { era, not } \\
\text { explained by } \\
\text { demographic or } \\
\text { socioeconomic } \\
\text { factors such as } \\
\text { increased } \\
\text { financial hardship } \\
\text { over time }\end{array}$ \\
\hline $\begin{array}{l}\text { (Oskroch } \\
\text { i et al., } \\
\text { 2018) }\end{array}$ & 2018 & $\begin{array}{l}\text { Cross } \\
\text { sectional }\end{array}$ & $\begin{array}{l}\text { Data from the } \\
\text { British } \\
\text { Household Panel } \\
\text { Survey (BHPS) } \\
\text { and the } \\
\text { Understanding } \\
\text { Society Survey } \\
\text { (USS. }\end{array}$ & $\begin{array}{l}\text { General Health } \\
\text { Questionnaire } \\
\text { (GHQ) }\end{array}$ & $\begin{array}{l}\text { British } \\
\text { Household } \\
\text { Panel Survey } \\
\text { (BHPS) and } \\
\text { Understandin } \\
\text { g Society (US) }\end{array}$ & $\begin{array}{l}\text { Clear effects } \\
\text { on both men } \\
\text { and } \\
\text { women's } \\
\text { well-being } \\
\text { as the head } \\
\text { of a } \\
\text { household } \\
\text { have been } \\
\text { noticed. }\end{array}$ & $\begin{array}{l}\text { financial status } \\
\text { has a significant } \\
\text { impact on the } \\
\text { psychological } \\
\text { wellbeing. }\end{array}$ \\
\hline $\begin{array}{l}\text { (Richard } \\
\text { son et } \\
\text { al., } \\
2017 \text { ) }\end{array}$ & 2017 & $\begin{array}{l}\text { Cross } \\
\text { sectional }\end{array}$ & $\begin{array}{l}\text { A longitudinal } \\
\text { design was used } \\
\text { to evaluate } \\
\text { whether financial } \\
\text { variables } \\
\text { influence mental } \\
\text { health overtime } \\
\text { changes in } \\
\text { undergraduates }\end{array}$ & (CORE-GP) & $\begin{array}{l}\text { Index of } \\
\text { Financial } \\
\text { Stress (IFS) }\end{array}$ & $\begin{array}{l}\text { Financial } \\
\text { difficulties } \\
\text { seem to } \\
\text { cause poor } \\
\text { mental } \\
\text { health in } \\
\text { students } \\
\text { with the } \\
\text { prospect of } \\
\text { a vicious } \\
\text { cycle. }\end{array}$ & $\begin{array}{l}\text { Financial factors } \\
\text { tend to lead to } \\
\text { poor mental } \\
\text { health rather than } \\
\text { mental health } \\
\text { issues. More } \\
\text { significant } \\
\text { financial } \\
\text { problems cross- } \\
\text { sectionally } \\
\text { expected greater } \\
\text { depression and } \\
\text { stress and } \\
\text { predicted worse } \\
\text { anxiety. }\end{array}$ \\
\hline
\end{tabular}




\begin{tabular}{|c|c|c|c|c|c|c|c|}
\hline Authors & Year & $\begin{array}{c}\text { Study } \\
\text { Design }\end{array}$ & Method & Mental Health & $\begin{array}{c}\text { Financial } \\
\text { Well - Being }\end{array}$ & Conclusion & $\begin{array}{l}\text { Relationship } \\
\text { between } \\
\text { Financial } \\
\text { Wellbeing and } \\
\text { Mental Health } \\
\text { issues }\end{array}$ \\
\hline $\begin{array}{l}\text { (Archulet } \\
\text { a et al., } \\
2019)\end{array}$ & 2019 & $\begin{array}{l}\text { Cross } \\
\text { sectional }\end{array}$ & $\begin{array}{l}\text { The financial } \\
\text { clinic used } \\
\text { quasi- } \\
\text { experimental } \\
\text { approaches as } \\
\text { both } \\
\text { A Financial } \\
\text { Planning and } \\
\text { Financial } \\
\text { Counseling } \\
\text { Research and } \\
\text { Training Clinic }\end{array}$ & $\begin{array}{l}\text { Financial } \\
\text { Anxiety Scale } \\
\text { (FAS) }\end{array}$ & $\begin{array}{l}\text { Solution- } \\
\text { Focused } \\
\text { Financial } \\
\text { Therapy } \\
\text { (SFFT) }\end{array}$ & $\begin{array}{l}\text { Results } \\
\text { indicate that } \\
\text { Solution- } \\
\text { Focused } \\
\text { Financial } \\
\text { Therapy } \\
\text { (SFFT) } \\
\text { approach to } \\
\text { targeting } \\
\text { can reduce } \\
\text { short-term } \\
\text { financial } \\
\text { anxiety. } \\
\text { Implications } \\
\text { for mental } \\
\text { wellbeing, } \\
\text { financial, } \\
\text { and study } \\
\text { therapy } \\
\text { practice are } \\
\text { formed. }\end{array}$ & $\begin{array}{l}\text { Financial anxiety } \\
\text { tends to have a } \\
\text { significant } \\
\text { influence on the } \\
\text { lives of many } \\
\text { Americans (APA } \\
\text { Stress in } \\
\text { America) }\end{array}$ \\
\hline $\begin{array}{l}\text { (Chaves } \\
\text { et al., } \\
2018 \text { ) }\end{array}$ & 2018 & & $\begin{array}{l}\text { The current } \\
\text { study examines } \\
\text { data collected in } \\
\text { Spain from } \\
\text { Round } 3 \text { (R3, } \\
2006 / 2007) \text { and } \\
\text { Round } 6 \text { (R6, } \\
2013 \text { ) of the ESS }\end{array}$ & $\begin{array}{l}\text { Depression } \\
\text { Scale (CESD8, } \\
\text { Radloff } \\
\\
\end{array}$ & \begin{tabular}{|l} 
Life \\
satisfaction
\end{tabular} & $\begin{array}{l}\text { Economic } \\
\text { crises are } \\
\text { associated } \\
\text { with } \\
\text { increased } \\
\text { depressive } \\
\text { symptoms. }\end{array}$ & $\begin{array}{l}\text { Financial crises } \\
\text { tend to have a } \\
\text { corrosive impact } \\
\text { on mental } \\
\text { wellbeing by } \\
\text { reducing the } \\
\text { mediating } \\
\text { influence of } \\
\text { positive values on } \\
\text { a good society. }\end{array}$ \\
\hline $\begin{array}{l}\text { (Thinaga } \\
\text { ran et } \\
\text { al., } \\
2017 \text { ) }\end{array}$ & 2017 & $\begin{array}{l}\text { Cross } \\
\text { sectional }\end{array}$ & $\begin{array}{l}\text { The survey } \\
\text { consists of } 1064 \\
\text { respondents } \\
\text { from low-cost } \\
\text { flats whose } \\
\text { household } \\
\text { income is below } \\
\text { RM3000 from } \\
\text { the Kuala } \\
\text { Lumpur City Hall } \\
\text { (DBKL) list using } \\
\text { a purposeful } \\
\text { sampling method } \\
\text { (n= 1064) }\end{array}$ & $\begin{array}{l}\text { Financial Well- } \\
\text { Being }\end{array}$ & $\begin{array}{l}\text { Financial } \\
\text { strains }\end{array}$ & $\begin{array}{l}\text { Financial } \\
\text { behavior } \\
\text { has a } \\
\text { positive } \\
\text { impact, and } \\
\text { financial } \\
\text { strain } \\
\text { negatively } \\
\text { affects the } \\
\text { urban poor } \\
\text { community's } \\
\text { well-being. }\end{array}$ & $\begin{array}{l}\text { The financial } \\
\text { strain affects the } \\
\text { urban poor } \\
\text { community's well- } \\
\text { being }\end{array}$ \\
\hline
\end{tabular}




\begin{tabular}{|c|c|c|c|c|c|c|c|}
\hline Authors & Year & $\begin{array}{l}\text { Study } \\
\text { Design }\end{array}$ & Method & Mental Health & $\begin{array}{c}\text { Financial } \\
\text { Well - Being }\end{array}$ & Conclusion & $\begin{array}{l}\text { Relationship } \\
\text { between } \\
\text { Financial } \\
\text { Wellbeing and } \\
\text { Mental Health } \\
\text { issues }\end{array}$ \\
\hline $\begin{array}{l}\text { (Viseu et } \\
\text { al., } \\
2018)\end{array}$ & 2018 & $\begin{array}{l}\text { Cross } \\
\text { sectional }\end{array}$ & $\begin{array}{l}\text { The sample of } \\
\text { this study } \\
\text { consisted of } 729 \\
\text { Portuguese } \\
\text { participants, } 247 \\
(33.9 \%) \text { males, } \\
\text { and } 482(66.1 \%) \\
\text { female structural } \\
\text { equation } \\
\text { modeling (SEM) } \\
\text { was used to ex- } \\
\text { amine the } \\
\text { defined } \\
\text { relationships. } \\
\text { (n= 729) }\end{array}$ & $\begin{array}{l}\text { Depression } \\
\text { Anxiety Stress } \\
\text { Scale 21-item } \\
\text { version } \\
\text { (DASS-21) }\end{array}$ & $\begin{array}{l}\text { Financial well- } \\
\text { being was } \\
\text { assessed by } \\
\text { the Financial } \\
\text { Well-Being } \\
\text { Scale (FWBS) }\end{array}$ & $\begin{array}{l}\text { The sample } \\
\text { collected does } \\
\text { not represent } \\
\text { the } \\
\text { Portuguese } \\
\text { population } \\
\text { Economic } \\
\text { stressors } \\
\text { (economic } \\
\text { hardship, } \\
\text { financial risk, } \\
\text { and financial } \\
\text { well-being) } \\
\text { relate } \\
\text { positively to } \\
\text { stress, } \\
\text { anxiety, and } \\
\text { depression. }\end{array}$ & $\begin{array}{l}\text { Statistically } \\
\text { significant } \\
\text { variations have } \\
\text { been identified in } \\
\text { the relationship } \\
\text { between financial } \\
\text { threat and } \\
\text { depression and } \\
\text { between } \\
\text { economic } \\
\text { hardship and } \\
\text { anxiety, anxiety, } \\
\text { and depression }\end{array}$ \\
\hline $\begin{array}{l}\text { (Bowers } \\
\text { et al., } \\
2018 \text { ) }\end{array}$ & 2018 & $\begin{array}{l}\text { Cross } \\
\text { sectional }\end{array}$ & $\begin{array}{l}\text { The survey was } \\
\text { conducted by } \\
1124 \text { workers at } \\
\text { remote } \\
\text { construction and } \\
\text { open cut and } \\
\text { underground } \\
\text { mining sites. } \\
\text { ( } \mathbf{n = 1 1 2 4 )}\end{array}$ & $\begin{array}{l}\text { The Kessler } \\
\text { Psychological } \\
\text { Distress Scale } \\
\text { questionnaire } \\
\text { (K10) }\end{array}$ & $\begin{array}{l}\text { financial } \\
\text { situation }\end{array}$ & $\begin{array}{l}\text { Psychological } \\
\text { distress is } \\
\text { significantly } \\
\text { more } \\
\text { prevalent in } \\
\text { remote mining } \\
\text { and } \\
\text { construction } \\
\text { workers than } \\
\text { in the overall } \\
\text { Australian } \\
\text { population. } \\
\text { Factors that } \\
\text { contribute to } \\
\text { mental illness } \\
\text { among these } \\
\text { workers need } \\
\text { to be explored } \\
\text { and stigma } \\
\text { associated } \\
\text { with mental } \\
\text { health issues } \\
\text { minimized }\end{array}$ & $\begin{array}{l}\text { People who } \\
\text { reported stress } \\
\text { due to the } \\
\text { stigmatization of } \\
\text { mental health } \\
\text { problems were at } \\
\text { the highest risk of } \\
\text { high/very high } \\
\text { psychological } \\
\text { distress. }\end{array}$ \\
\hline $\begin{array}{l}\text { (M. } \\
\text { Taylor et } \\
\text { al., } \\
2017 \text { ) }\end{array}$ & 2017 & $\begin{array}{l}\text { Cross } \\
\text { sectional }\end{array}$ & $\begin{array}{l}\text { This study } \\
\text { investigated } \\
\text { psychological } \\
\text { distress among } \\
\text { parents in } \\
\text { Western Sydney } \\
\text { households } \\
(\mathbf{n = 4 3 9 )}\end{array}$ & $\begin{array}{l}\text { Kessler } 10 \\
\text { Measure of } \\
\text { Psychological } \\
\text { Distress (K10). }\end{array}$ & $\begin{array}{l}\text { Income and } \\
\text { Labour } \\
\text { Dynamics in } \\
\text { Australia } \\
\text { (HILDA) }\end{array}$ & $\begin{array}{l}\text { Financial } \\
\text { hardship } \\
\text { factors formed } \\
\text { the strongest } \\
\text { associations } \\
\text { of reported } \\
\text { psychological } \\
\text { distress } \\
\text { among } \\
\text { Western } \\
\text { Sydney } \\
\text { parents, } \\
\text { especially } \\
\text { those related } \\
\text { to } \\
\text { mortgage/hou } \\
\text { sing and job } \\
\text { security. }\end{array}$ & $\begin{array}{l}\text { Multivariate } \\
\text { analysis showed } \\
\text { that financial } \\
\text { hardship factors } \\
\text { influenced the } \\
\text { strongest } \\
\text { psychological } \\
\text { distress } \\
\text { correlations }\end{array}$ \\
\hline
\end{tabular}




\begin{tabular}{|c|c|c|c|c|c|c|c|}
\hline Authors & Year & $\begin{array}{l}\text { Study } \\
\text { Design }\end{array}$ & Method & Mental Health & $\begin{array}{c}\text { Financial } \\
\text { Well - Being }\end{array}$ & Conclusion & $\begin{array}{l}\text { Relationship } \\
\text { between } \\
\text { Financial } \\
\text { Wellbeing and } \\
\text { Mental Health } \\
\text { issues }\end{array}$ \\
\hline $\begin{array}{l}\text { (Kiely et } \\
\text { al., } \\
2015 \text { ) }\end{array}$ & 2015 & $\begin{array}{l}\text { Cross } \\
\text { sectional }\end{array}$ & $\begin{array}{l}\text { Income and } \\
\text { Labour } \\
\text { Dynamics in } \\
\text { Australia } \\
\text { (HILDA) survey. } \\
\text { (n= 11,134) }\end{array}$ & $\begin{array}{l}\text { Mental Health } \\
\text { Inventory } \\
(\mathrm{MHI}-5)\end{array}$ & $\begin{array}{l}\text { Financial } \\
\text { hardship }\end{array}$ & $\begin{array}{l}\text { Existing } \\
\text { hardship } \\
\text { was linked } \\
\text { to increased } \\
\text { mental } \\
\text { health risk. } \\
\text { This was } \\
\text { beyond } \\
\text { those } \\
\text { exposed to } \\
\text { hardship } \\
\text { and } \\
\text { deprivation's } \\
\text { poorer } \\
\text { mental } \\
\text { health. }\end{array}$ & $\begin{array}{l}\text { Individuals with } \\
\text { difficulties at any } \\
\text { stage in the } \\
\text { research had } \\
\text { higher rates of } \\
\text { mental health } \\
\text { issues than those } \\
\text { never subject to } \\
\text { struggle }\end{array}$ \\
\hline $\begin{array}{l}\text { (Furey et } \\
\text { al., } \\
2016) \text {. }\end{array}$ & 2016 & $\begin{array}{l}\text { Cross } \\
\text { sectional }\end{array}$ & $\begin{array}{l}\text { All participants } \\
\text { have involved } \\
\text { farmers }(n=121) \\
\text { aged 18-80. } \\
\text { The 45- to 54- } \\
\text { year-old group } \\
\text { (33.9\%, } n=41) \\
\text { was the most } \\
\text { common age } \\
\text { group. Ethnicity } \\
\text { included Euro- } \\
\text { Caucasians } \\
\text { (n= 121). }\end{array}$ & $\begin{array}{l}\text { Patient Health } \\
\text { Questionnaire } \\
\text { (PHQ-8) } \\
\text { Anxiety } \\
\text { Disorder } \\
\text { Assessment } \\
\text { (GAD 7). }\end{array}$ & $\begin{array}{l}\text { Financial } \\
\text { Threat Scale } \\
\text { (FTS) }\end{array}$ & $\begin{array}{l}\text { Mental } \\
\text { distress } \\
\text { directly } \\
\text { effects of } \\
\text { non- } \\
\text { financial } \\
\text { farm stress } \\
\text { (FS) } \\
\text { approached } \\
\text { significance. } \\
\text { Mental } \\
\text { distress } \\
\text { mediated } \\
\text { these } \\
\text { relationships } \\
\text { as } \\
\text { evidenced } \\
\text { by } \\
\text { significant } \\
\text { indirect } \\
\text { effects of } \\
\text { farm stress } \\
\text { (FS) and } \\
\text { financial } \\
\text { threat (FT), } \\
\text { and Social } \\
\text { Support } \\
\text { served to } \\
\text { reduce } \\
\text { distress. }\end{array}$ & $\begin{array}{l}\text { Financial threat } \\
(\mathrm{FT}), \text { mental } \\
\text { health concerns } \\
\text { prevention is } \\
\text { better than cure } \\
\text { from both an } \\
\text { economic and a } \\
\text { social justice } \\
\text { perspective. }\end{array}$ \\
\hline
\end{tabular}




\begin{tabular}{|c|c|c|c|c|c|c|c|}
\hline Authors & Year & $\begin{array}{l}\text { Study } \\
\text { Design }\end{array}$ & Method & Mental Health & $\begin{array}{c}\text { Financial } \\
\text { Well - Being }\end{array}$ & Conclusion & $\begin{array}{c}\text { Relationship } \\
\text { between } \\
\text { Financial } \\
\text { Wellbeing and } \\
\text { Mental Health } \\
\text { issues }\end{array}$ \\
\hline $\begin{array}{l}\text { (Econom } \\
\text { ou et al., } \\
2018)\end{array}$ & 2018 & $\begin{array}{l}\text { Qualitativ } \\
\text { e } \\
\text { variables } \\
\text { are } \\
\text { presente } \\
\text { d with } \\
\text { absolute } \\
\text { and } \\
\text { relative } \\
\text { frequenci } \\
\text { es }\end{array}$ & $\begin{array}{l}\text { A random and } \\
\text { representative } \\
\text { sample of } 600 \\
\text { adult Cypriots } \\
\text { participated in } \\
\text { the study by } \\
\text { attempting to } \\
\text { explore the } \\
\text { prevalence and } \\
\text { correlates of } \\
\text { major } \\
\text { depression in } \\
\text { Cyprus during } \\
\text { the financial } \\
\text { crisis. } \\
\text { (n= 600) }\end{array}$ & $\begin{array}{l}\text { Structured } \\
\text { Clinical } \\
\text { Interview } \\
\text { (SCl-D) }\end{array}$ & $\begin{array}{l}\text { Index of } \\
\text { Personal } \\
\text { Economical } \\
\text { Distress } \\
\text { (IPED) }\end{array}$ & $\begin{array}{l}\text { Financial } \\
\text { hardship } \\
\text { was found } \\
\text { to be a risk } \\
\text { factor for } \\
\text { major } \\
\text { depression, } \\
\text { while social } \\
\text { networks } \\
\text { and } \\
\text { institutional } \\
\text { trust were } \\
\text { found to be } \\
\text { protective. }\end{array}$ & $\begin{array}{l}\text { The impact of the } \\
\text { financial crisis on } \\
\text { mental health is } \\
\text { unequally } \\
\text { distributed } \\
\text { among Cyprus' } \\
\text { general } \\
\text { population. }\end{array}$ \\
\hline $\begin{array}{l}\text { (Wan et } \\
\text { al., } \\
2019 \text { ) }\end{array}$ & 2019 & $\begin{array}{l}\text { Hierarchi } \\
\text { cal } \\
\text { regressio } \\
n\end{array}$ & $\begin{array}{l}\text { A stratified } \\
\text { sample of CAF } \\
\text { participants from } \\
\text { three } \\
\text { representative } \\
\text { bases } \\
\text { (Petawawa, } \\
\text { Halifax, and Cold } \\
\text { Lake; } N=4,700 \text { ) } \\
\text { was sent to } \\
\text { complete the } \\
\text { online Military } \\
\text { Group Wellness. } \\
\text { (n= 4,700) }\end{array}$ & $\begin{array}{l}\text { Kessler } \\
\text { Psychological } \\
\text { Distress Scale } \\
\text { (K10) }\end{array}$ & $\begin{array}{l}\text { Overall Life } \\
\text { Satisfaction } \\
\text { Scale }\end{array}$ & $\begin{array}{l}\text { Financial } \\
\text { satisfaction } \\
\text { is a } \\
\text { significant } \\
\text { indicator of } \\
\text { life } \\
\text { satisfaction } \\
\text { and } \\
\text { psychologic } \\
\text { al distress } \\
\text { among } \\
\text { members of } \\
\text { the } \\
\text { Canadian } \\
\text { Armed } \\
\text { Forces } \\
\text { (CAF). }\end{array}$ & $\begin{array}{l}\text { Financial } \\
\text { satisfaction } \\
\text { expected } \\
\text { psychological } \\
\text { distress. }\end{array}$ \\
\hline $\begin{array}{l}\text { (Selenko } \\
\& \\
\text { Batinic, } \\
2011 \text { ) }\end{array}$ & 2011 & $\begin{array}{l}\text { Cross } \\
\text { sectional }\end{array}$ & $\begin{array}{l}\text { Clients of a } \\
\text { national non- } \\
\text { profit, publicly } \\
\text { funded Austrian } \\
\text { debt-counseling } \\
\text { institution facing } \\
\text { bankruptcy filing. } \\
\text { Their counselors } \\
\text { met them in } \\
\text { Spring } 2009 \\
(n=106)\end{array}$ & $\begin{array}{l}\text { General Health } \\
\text { Questionnaire }\end{array}$ & financial strain & $\begin{array}{l}\text { The results } \\
\text { show the } \\
\text { importance } \\
\text { of subjective } \\
\text { economic } \\
\text { stress in } \\
\text { predicting } \\
\text { mental } \\
\text { health } \\
\text { among } \\
\text { persons } \\
\text { under } \\
\text { severe } \\
\text { financial } \\
\text { pressure } \\
\text { and indicate } \\
\text { essential } \\
\text { moderators. }\end{array}$ & $\begin{array}{l}\text { The findings } \\
\text { show the } \\
\text { importance of } \\
\text { subjective } \\
\text { economic stress } \\
\text { on predicting } \\
\text { mental health } \\
\text { among people } \\
\text { under severe } \\
\text { financial strain } \\
\text { and point to } \\
\text { important } \\
\text { moderators of } \\
\text { this relationship. }\end{array}$ \\
\hline
\end{tabular}




\begin{tabular}{|c|c|c|c|c|c|c|c|}
\hline Authors & Year & $\begin{array}{l}\text { Study } \\
\text { Design }\end{array}$ & Method & Mental Health & $\begin{array}{c}\text { Financial } \\
\text { Well - Being }\end{array}$ & Conclusion & $\begin{array}{l}\text { Relationship } \\
\text { between } \\
\text { Financial } \\
\text { Wellbeing and } \\
\text { Mental Health } \\
\text { issues }\end{array}$ \\
\hline $\begin{array}{l}\text { (M. P. } \\
\text { Taylor et } \\
\text { al., } \\
2011)\end{array}$ & 2011 & $\begin{array}{l}\text { Prospecti } \\
\text { ve cohort } \\
\text { ( } 2 \\
\text { waves) }\end{array}$ & $\begin{array}{l}\text { They were using } \\
\text { National } \\
\text { Representative } \\
\text { Survey. Data } \\
\text { from the British } \\
\text { Household } \\
\text { Survey } 1991- \\
2006 \text { was used } \\
\text { to build a } \\
\text { measure of } \\
\text { financial ability. }\end{array}$ & $\begin{array}{l}\text { General Health } \\
\text { Questionnaire }\end{array}$ & $\begin{array}{l}\text { Data from the } \\
\text { 1991-2006 } \\
\text { British } \\
\text { Household } \\
\text { Panel Survey } \\
\text { was used to } \\
\text { measure } \\
\text { financial } \\
\text { capacity. }\end{array}$ & $\begin{array}{l}\text { The } \\
\text { financial } \\
\text { potential } \\
\text { has } \\
\text { significant } \\
\text { psychologic } \\
\text { al health } \\
\text { consequenc } \\
\text { es. }\end{array}$ & $\begin{array}{l}\text { The effect of } \\
\text { having low } \\
\text { financial capacity } \\
\text { is known to be a } \\
\text { significant } \\
\text { determinant of } \\
\text { mental illness, } \\
\text { with low financial } \\
\text { capacity } \\
\text { worsening } \\
\text { psychological } \\
\text { costs. }\end{array}$ \\
\hline $\begin{array}{l}\text { (Marjano } \\
\text { vic et al., } \\
2013 \text { ) }\end{array}$ & 2013 & $\begin{array}{l}\text { Cross } \\
\text { sectional }\end{array}$ & $\begin{array}{l}\text { Participants } \\
\text { were } \\
\text { undergraduate } \\
\text { psychology } \\
\text { ( } n=292) \text { and } \\
\text { business } \\
\text { students ( } n=188) \\
\text { who completed a } \\
\text { 30-minute online } \\
\text { questionnaire as } \\
\text { part of a broader } \\
\text { inquiry into an } \\
\text { economic } \\
\text { downturn and } \\
\text { mental health. } \\
\text { (n= 480) }\end{array}$ & $\begin{array}{l}\text { General Health } \\
\text { Questionnaire }\end{array}$ & $\begin{array}{l}\text { Economic } \\
\text { Hardship } \\
\text { Scale }\end{array}$ & $\begin{array}{l}\text { Studies } \\
\text { have shown } \\
\text { that the FTS } \\
\text { is useful as } \\
\text { both a } \\
\text { psychologic } \\
\text { al indicator, } \\
\text { and perhaps } \\
\text { its most } \\
\text { significant } \\
\text { advantage } \\
\text { over other } \\
\text { indicators is } \\
\text { its general } \\
\text { ability. }\end{array}$ & $\begin{array}{l}\text { The Financial } \\
\text { Threat Scale } \\
\text { (FTS) also } \\
\text { correlated with all } \\
\text { the predicted } \\
\text { psychological } \\
\text { health } \\
\text { interventions. It } \\
\text { was positively } \\
\text { associated with } \\
\text { psychological } \\
\text { distress and } \\
\text { mood } \\
\text { disturbance in } \\
\text { combination with } \\
\text { the current } \\
\text { financial } \\
\text { situation. }\end{array}$ \\
\hline
\end{tabular}

Key: 1 . The research question or objective in this paper clearly stated.

2. The study design and study population clearly defined.

3. The tool of mental health and financial well-being clearly stated.

4. The Independent Variables clearly defined, valid and reliable to all study participants.

5. The Dependent Variables clearly defined, valid and reliable to all study participants.

\subsection{Summary of Evidence}

Overall, this study found 32 papers exploring relationships between financial well-being and mental health. Findings have varied by assessing methods used for each financial well-being and mental wellbeing. 4 Longitudinal experiments were used to examine the relationship between variables. The bidirectional relationship between financial problems and mental health was minimal.

Most of the findings shown that financial well-being has a significant impact on mental health. This systematic study uses many methods to assess financial well-being and mental health. Tool selection depends on research methodology and objectives. Evidence of financial hardship, the financial burden on mental health was more consistent. The financial well-being of mental health is also used as a measure of stress, anxiety and depression. Most studies have shown that financial hardship is closer to depression.

Greater financial difficulties predicted for increased depression and stress, and also predicted for poorer anxiety. Besides, five studies have indicated that the economic downturn impacts have also contributed to mental health concerns, which have been positively related to a substantial rise in depressive symptoms. A substantial rise in symptoms of depression and anxiety was found during the GFC (Global Finance Crisis). On the negative side, the recession impacted individuals' financial wellbeing and had adverse mental health effects. To summarize, it was found that the mental health aspect was greatly affected by the financial situation. 


\subsection{Financial Wellbeing Measurement Tools}

The analysis showed most of the studies used cross-sectional for the design of the research study. Most of the quantitative research contrasted and used less qualitatively. In a cross-section, the researcher analyses the outcome and exposures of the study participants simultaneously, particularly from financial well-being and assessment of mental well-being. From the analysis, it could be said that most cross-section studies are easier to collect data and can be easily measured and applied to the target populations; on the other hand, they are easier to execute and more managed. In several reports, financial well-being and assessment of mental health over different periods, financial distress was assessed by asking the respondent to gather information from the measurement of financial wellbeing.

From the measurement tools in this study review, there is an evidence on the relationship between financial strain and mental health. (Aranda \& Lincoln, 2011) defined that the financial strain is a chronic economic stressor that can have especially adverse effects on an individual's well-being. The findings stated the financial strain increased the level of depressive symptoms among this sample. It is align with (Zürcher et al., 2019) whereby prove that the financial strain was associated with general mental health problems and depressive symptomatology. Meanwhile for others study that using financial strain in financial well-being measurement conducted by (Thinagaran et al., 2017) which is the data was obtained by using self-administered questionnaires and the data being analyzed by descriptive analysis meanwhile, and multi regression analysis shows that financial strain has a negative influence on the personal well-being of the poor urban community. (Selenko \& Batinic, 2011) indicated there is a significant negative effect of perceived financial strain on mental health. Hence, it could be concluded financial strain has a major effect on mental health even if the study setting differs for each setting.

The study examined the association between economic stress factors and stress, anxiety, and depression, as well as the moderating effect of social support using financial well-being measured by the financial Well-Being Scale (FWBS) (Viseu et al., 2018); (Norvilitis et al., 2003) With the eight-point Likert scale (1- Strongly disagree; 5-Strongly agree) argument (e.g., I am uncomfortable with the amount of debt I am in). The value of the Variance Derived (AVE) is 0.66 . This study presents some considerable limitations. The collected sample may not represent the Portuguese population involved and comprises 729 Portuguese participants, 247 (33.9\%) males, and 482 (66.1\%) females. Nevertheless, their composition is rather unbalanced. The number of working persons constitutes $80 \%$ of the overall sample, while $20 \%$ comprises unemployed or retired persons.

Therefore, a comparison will often favor the group employed to the detriment of the other two groups. However, the results may be adapted. It could be useful to perform parallel research in other countries about how the mental health factor can affect adverse financial well-being situations. (Economou et al., 2018) used Index of Personal Economic Distress (IPED) analysis as to evaluate the degree of the financial strain of the participants. As an overview, IPED is the instrument used to assess financial well-being. The tools are an initial, self-reported scale that affects participants' difficulty managing financial needs over the last 6 months. Activities shall be calculated as to the payment of a loan/credit card bill, monthly rent, taxes. IPED included 8 items, responses on a 3 -point scale $(1=$ never, $2=$ often, and $3=$ often and a composite score of ' 15 and above' suggested high financial distress. The internal consistency of the scale was found to be strong (Cronbach $a=0.77$ ). From the results of financial strain elements, using IPED, paying household bills is the strongest association with major depression in univariate analysis.

Overall, people who sometimes/often experience difficulties in paying these expenses showed a significantly higher risk of severe depression than those who rarely/never face these difficulties or still have no expenses. Only rent payment was not correlated with major depression. Another research using IPED as a financial well-being measurement (Economou et al., 2013) was performed using the Two national cross-sectional telephone surveys methods in 2008 and 2011 in the same methodology. The particular self-reported measure encompasses eight questions illustrating the difficulty of 
participants meeting a household's daily financial demands (from loan payments to grocery payments) over the past six months. Responses are rendered on a three-point scale, representing frequency dimensions: never (1), often (2), and sometimes (3), while the composite scale will vary from 8 (no economic problems) to 24 (serious economic problems). A significant correlation was noticed between major depression and economic hardship. (Economou et al., 2016). Meanwhile, a stratified sampling technique for 2,188 citizens was performed to estimate the incidence of major depression. Participants' degree of financial difficulty was assessed by the Personal Economic Distress Index (IPED) and found that unemployment and economic hardship were found to increase the probability of suffering from major depression.

On the other hand, Financial well-being tools with a reliable financial treatment scale (FTS) (Cronbach's $\alpha=0.89$ ). FTS has proven reliability. The FTS was developed based on current threat and threat research to evaluate hypothesized FT. The items represent areas related to (i) risk threat (e.g., "How much do you feel at risk?"); (ii) threat-related concerns; (iii) anticipated threat; (iv) mental fixation on individual personal finances, and (v) threat uncertainty. For each element, participants responded on a five-point scale ranging from 1 (Not at all) to 5 (Extremely), indicating the accuracy of statements representing their personal feelings about current FTs.

Participants completed the entire FTS scale of five items. Two items from the Family, Income, and Labor Dynamics in Australia (HILDA) survey to measure financial well-being were analyzed in other environments (M. Taylor et al., 2017) in a study using household financial pressure. A random sample of adults aged over 18 years) living in the western suburbs of Sydney, NSW, where the research participants. Variables of financial stressors were independently examined, with variables of individual and community support significantly correlated with increased psychological distress.

\subsection{Mental Health Measurement Tools}

From this Systematic Literature Reviews, General Health Questionnaire - 12 utilized by (Marjanovic et al., 2013) GHQ-12 is a commonly used, brief measure of psychological distress issues such as depression, anxiety, social functioning, and confidence. In this setting research, undergraduate psychology students $(n=292)$ and business students $(n=188)$ completed a 30-minute online questionnaire as part of a wider investigation into an economic downturn and mental health. The findings revealed a positive correlation with psychological distress (i.e., GHQ-12 reverse score; $r=.38$, $p<.001)$, depression $(r=.36, p<.001)$, anxiety $(r=.34, p<.001)$, and mood disturbance related to one's current financial situation ( $r=.65$, $p<.001$ ). (M. P. Taylor et al., 2011) Using the 12 -item General Health Questionnaire, respondents' psychological health results show that those with high financial capacity experienced a decrease in their GHQ scores. In contrast, those with low financial capacity experienced GHQ scores.

Financial status and male gender have long been associated with mental-wellbeing scores supported by the study (Oskrochi et al., 2018) that men with low financial capacity affect mental health. In comparison (Lewko et al., 2019), using the General health questionnaire (GHQ-28) as a screening tool to assess the existence of mental illness and allow the different mental health populations studied. The mental health component was found to have a significant financial influence $(p=0.005)$. The types of General Health Questionnaire used to rely on the study's objective and setting to use GHQ-12 or (GHQ-28).

To conclude, all findings that specifically addressed that financial capacity is positively associated with psychological wellbeing. GHQ-12 used in this study is a simple, reliable, and responsive short form-ideal for research studies. However, this measure is not precise in many works of literature to evaluate the degree, different types of mental illness compare Depression Anxiety Stress Scale 21-item version (DASS-21) that the most accurate and useful for evaluating common mental health symptoms is especially depression, anxiety, and stress, and the three dimensions of DASS-21 consists of twentyone items. DASS-21 measures depression, stress, and anxiety. These three elements. Depression Anxiety Stress Scales (DASS) were translated. Moreover, validated in different languages in different 
communities. DASS's original version consists of 42 objects. However, the latest version has been adjusted as it is best used for either clinical or non-clinical settings (Crawford \& Henry, 2003). Reason for using the DASS-21 questionnaire for mental health measurements, as the questionnaire items are simple and easy to understand by the respondents, as there is no training required compared to other Psychometric research. Using DASS-21, researchers will concurrently assess stress, anxiety, and depression levels. All of the cultural-free question in the DASS-21, not one of the objects, is listed or linked to any culture or religion. Therefore, DASS-21 will be used to assess correspondence mental wellbeing.

\section{Conclusion}

The results reveal that from systematically reviewing the literature in the context of financial hardship and mental health, the effect of psychological variables was examined in order to decide which variables the mechanisms are most frequently and reliably concerned. The findings also revealed that in some studies, these variables had been considered. This review also involves studies of various designs conducted with a variety of populations from around the world, in the context of financial hardship, psychological variables associated with mental health. This is important to the next future direction as it will help the researchers, policymakers to strategies for the next exploration within the same area.

\section{Acknowledgement}

We would like to express our sincere gratitude Faculty of Business and Management, Universiti Teknologi MARA Selangor, Puncak Alam Campus, for their kind support and contributions. This study was funded by Universiti Teknologi MARA via GIP Grant: 600-IRMI 5/3/GIP (013/2019).

\section{References}

1. Alyousef, S. M. (2019). Psychosocial stress factors among mental health nursing students in KSA. Journal of Taibah University Medical Sciences, 14(1), 60-66.

2. Anderson, M., Revie, C. W., Quail, J. M., Wodchis, W., de Oliveira, C., Osman, M., Baetz, M., McClure, J., Stryhn, H., \& Buckeridge, D. (2018). The effect of socio-demographic factors on mental health and addiction high-cost use: a retrospective, population-based study in Saskatchewan. Canadian Journal of Public Health, 109(5), 810-820.

3. Antunes, A., Frasquilho, D., Zózimo, J. R., Silva, M., Cardoso, G., Ferrão, J., \& Caldas-de-Almeida, J. $M$. (2019). Solutions to tackle the mental health consequences of the economic recession: A qualitative study integrating primary health care users and professionals' perspectives. Health Policy, 123(12), 1267-1274.

4. Aranda, M. P., \& Lincoln, K. D. (2011). Financial strain, negative interaction, coping styles, and mental health among low-income Latinos. Race and Social Problems, 3(4), 280-297.

5. Archuleta, K. L., Asebedo, S. D., \& Palmer, L. (2019). Contemporary Theories and Frameworks for Use in Financial Counseling. In Financial Counseling (pp. 91-109). Springer.

6. Åslund, C., Larm, P., Starrin, B., \& Nilsson, K. W. (2014). The buffering effect of tangible social support on financial stress: influence on psychological well-being and psychosomatic symptoms in a large sample of the adult general population. International Journal for Equity in Health, 13(1), 85.

7. Bowers, J., Lo, J., Miller, P., Mawren, D., \& Jones, B. (2018). Psychological distress in remote mining and construction workers in Australia. Medical Journal of Australia, 208(9), 391-397.

8. Bureau, C. F. P. (2017). What is a payday loan. June.

9. Butterworth, P., Leach, L. S., Strazdins, L., Olesen, S. C., Rodgers, B., \& Broom, D. (2011). The psychosocial quality of work determines whether employment has benefits for mental health: results from a longitudinal national household panel survey. Occup Environ Med, 68(11), 806-812. 
10.Butterworth, P., Olesen, S. C., \& Leach, L. S. (2012). The role of hardship in the association between socio-economic position and depression. Australian \& New Zealand Journal of Psychiatry, 46(4), 364-373.

11.Chaves, C., Castellanos, T., Abrams, M., \& Vazquez, C. (2018). The impact of economic recessions on depression and individual and social well-being: the case of Spain (2006-2013). Social Psychiatry and Psychiatric Epidemiology, 53(9), 977-986.

12.De Witte, H., Vander Elst, T., \& De Cuyper, N. (2015). Job insecurity, health and well-being. In Sustainable working lives (pp. 109-128). Springer.

13.Economou, M., Angelopoulos, E., Peppou, L. E., Souliotis, K., Tzavara, C., Kontoangelos, K., Madianos, M., \& Stefanis, C. (2016). Enduring financial crisis in Greece: prevalence and correlates of major depression and suicidality. Social Psychiatry and Psychiatric Epidemiology, 51(7), 10151024.

14.Economou, M., Madianos, M., Peppou, L. E., Patelakis, A., \& Stefanis, C. N. (2013). Major depression in the era of economic crisis: a replication of a cross-sectional study across Greece. Journal of Affective Disorders, 145(3), 308-314.

15.Economou, M., Souliotis, K., Peppou, L. E., Agapidaki, I., Tzavara, C., \& Stefanis, C. N. (2018). Major depression in Cyprus amid financial crisis: prevalence and correlates. International Journal of Culture and Mental Health, 11(3), 255-267.

16.Esan, O. B., Kola, L., \& Gureje, O. (2012). Mental disorders and earnings: results from the Nigerian National Survey of Mental Health and Well-Being (NSMHW). The Journal of Mental Health Policy and Economics, 15(2), 77-82.

17.Fiksenbaum, L., Marjanovic, Z., \& Greenglass, E. (2017). Financial threat and individuals' willingness to change financial behavior. Review of Behavioral Finance. https://doi.org/10.1108/RBF-09-20160056

18.Furey, E. M., O'Hora, D., McNamara, J., Kinsella, S., \& Noone, C. (2016). The roles of Financial Threat, social support, Work stress, and Mental Distress in Dairy Farmers' expectations of injury. Frontiers in Public Health, 4, 126.

19.Gerrans, P., Speelman, C., \& Campitelli, G. (2014). The relationship between personal financial wellness and financial wellbeing: A structural equation modelling approach. Journal of Family and Economic Issues, 35(2), 145-160.

20.Greenglass, E. R., Katter, J. K. Q., Fiksenbaum, L., \& Hughes, B. M. (2015). Surviving in difficult economic times: relationship between economic factors, self-esteem and psychological distress in university students. In The Multi-generational and Aging Workforce. Edward Elgar Publishing.

21.Jian, J., Chen, C., \& Chen, F. (2014). Consumer financial capability and financial satisfaction. Social Indicators Research, 118(1), 415-432.

22.Jones, S. M., Chennupati, S., Nguyen, T., \& Fedorenko, C. R. (2018). Financial Burden Over Time in Older Adults with Cancer, Heart Disease, or Diabetes. Value in Health, 21, S145.

23.Kiely, K. M., Leach, L. S., Olesen, S. C., \& Butterworth, P. (2015). How financial hardship is associated with the onset of mental health problems over time. Social Psychiatry and Psychiatric Epidemiology, 50(6), 909-918.

24.Lewko, J., Misiak, B., \& Sierżantowicz, R. (2019). The Relationship between Mental Health and the Quality of Life of Polish Nurses with Many Years of Experience in the Profession: A Cross-Sectional Study. International Journal of Environmental Research and Public Health, 16(10), 1798.

25.Mandaliya, H., Ansari, Z., Evans, T., Oldmeadow, C., \& George, M. (2016). Psychosocial analysis of cancer survivors in rural Australia: focus on demographics, quality of life and financial domains. Asian Pac J Cancer Prev, 17(5), 2459-2464.

26.Marjanovic, Z., Greenglass, E. R., Fiksenbaum, L., \& Bell, C. M. (2013). Psychometric evaluation of the Financial Threat Scale (FTS) in the context of the great recession. Journal of Economic Psychology, 36, 1-10.

27.Mo, P. K. H., Cheng, Y., \& Lau, J. T. F. (2020). Work-related factors on mental health among migrant factory workers in china: Application of the Demand-Control and Effort-Reward Imbalance Model. Health \& Social Care in the Community. 
28.Mokhtar, N., Husniyah, A. R., Sabri, M. F., \& Abu Talib, M. (2015). Financial well-being among public employees in Malaysia: A preliminary study. Asian Social Science. https://doi.org/10.5539/ass.v11n18p49

29.Norvilitis, J. M., Szablicki, P. B., \& Wilson, S. D. (2003). Factors influencing levels of credit-card debt in College Students 1. Journal of Applied Social Psychology, 33(5), 935-947.

30.Organization, W. H. (2017). Depression and other common mental disorders: global health estimates. World Health Organization.

31.Oskrochi, G., Bani-Mustafa, A., \& Oskrochi, Y. (2018). Factors affecting psychological well-being: Evidence from two nationally representative surveys. PloS One, 13(6), e0198638.

32.Rajani, N. B., Giannakopoulos, G., \& Filippidis, F. T. (2016). Job insecurity, financial difficulties and mental health in Europe. Occupational Medicine, 66(8), 681-683.

33.Richardson, T., Elliott, P., Roberts, R., \& Jansen, M. (2017). A longitudinal study of financial difficulties and mental health in a national sample of British undergraduate students. Community Mental Health Journal, 53(3), 344-352.

34.Richardson, T., Jansen, M., \& Fitch, C. (2019). Financial difficulties in bipolar disorder part 2: psychological correlates and a proposed psychological model. Journal of Mental Health, 1-9.

35.Sabri, M. F., \& Zakaria, N. F. (2015). Financial Well-Being among Young Employees in Malaysia. https://doi.org/10.4018/978-1-4666-7484-4.ch013

36.Sargent-Cox, K., Butterworth, P., \& Anstey, K. J. (2011). The global financial crisis and psychological health in a sample of Australian older adults: a longitudinal study. Social Science \& Medicine, 73(7), 1105-1112.

37.Selenko, E., \& Batinic, B. (2011). Beyond debt. A moderator analysis of the relationship between perceived financial strain and mental health. Social Science \& Medicine, 73(12), 1725-1732.

38.Sturgeon, J. A., Arewasikporn, A., Okun, M. A., Davis, M. C., Ong, A. D., \& Zautra, A. J. (2016). The psychosocial context of financial stress: Implications for inflammation and psychological health. Psychosomatic Medicine, 78(2), 134.

39.Tanskanen, K., Ahola, T., Aminoff, A., Bragge, J., Kaipia, R., \& Kauppi, K. (2017). Towards evidencebased management of external resources: Developing design propositions and future research avenues through research synthesis. Research Policy, 46(6), 1087-1105.

40.Taylor, M. P., Jenkins, S. P., \& Sacker, A. (2011). Financial capability and psychological health. Journal of Economic Psychology, 32(5), 710-723.

41.Taylor, M., Stevens, G., Agho, K., \& Raphael, B. (2017). The Impacts of Household Financial Stress, Resilience, Social Support, and Other Adversities on the Psychological Distress of Western Sydney Parents. International Journal of Population Research, 2017.

42.Thinagaran, A., Dass, L. M., \& Sabri, M. F. (2017). The Financial Status and Factors Affecting Personal Well-Being Among Urban Poor in Kuala Lumpur. Malaysian Journal of Social Sciences and Humanities (MJSSH), 2(1), 21-39.

43.Tøge, A. G. (2016). Health effects of unemployment in Europe (2008-2011): a longitudinal analysis of income and financial strain as mediating factors. International Journal for Equity in Health, 15(1), 75.

44.Viseu, J., Leal, R., de Jesus, S. N., Pinto, P., Pechorro, P., \& Greenglass, E. (2018). Relationship between economic stress factors and stress, anxiety, and depression: Moderating role of social support. Psychiatry Research, 268, 102-107.

45.Wan, C., Berlinguette, M. K., \& Skomorovsky, A. (2019). The impact of financial satisfaction on wellbeing of Canadian military members. Journal of Military, Veteran and Family Health, 5(S1), 10-19.

46.Wilkinson, L. R. (2016). Financial strain and mental health among older adults during the great recession. Journals of Gerontology Series B: Psychological Sciences and Social Sciences, 71(4), 745754.

47.Zürcher, C., Tough, H., Fekete, C., \& Group, S. S. (2019). Mental health in individuals with spinal cord injury: The role of socioeconomic conditions and social relationships. PloS One, 14(2), e0206069. 